18

\title{
Theory of Frenkel Excitons in Planar Arrays of Perovskite Quantum Dots*
}

(C I.A. Vovk, N.V. Tepliakov, A.S. Baimuratov, M.Yu. Leonov, A.V. Baranov, A.V. Fedorov, and I.D. Rukhlenko Information Optical Technologies Center, ITMO University,

197101 St. Petersburg, Russia

e-mail: ilia.a.vovk@gmail.com

Received July 6, 2018

Of primary interest for the design of novel optoelectronic devices are densely packed ordered arrays of quantum dots (QDs), which can be produced via self-assembly or other nanofabrication techniques. In such arrays, also known as supercrystals, the interaction between the QDs results in a collective optical response. Despite the active research on arrays made of perovskite QDs, a rigorous theory of their collective excitations is still lacking. In this Paper, we develop a quantum-mechanical theory of Frenkel excitons in planar perovskite QD arrays with square lattice.

DOI: $10.21883 /$ OS.2018.11.46826.222-18

\footnotetext{
* International Conference „PCNSPA 2018 - Photonic Colloidal Nanostructures: Synthesis, Properties, and Applications", Saint Petersburg, Russia, June 4-8, 2018.

Полный текст статьи опубликован в английской версии журнала.
} 